\title{
Comparison of Analgesic Efficacy of Transversus Abdominis Plane Block Versus Infiltration of Local Anaesthetic into Surgical Wound In Emergency Laparotomies: A Randomized Control Trial
}

\section{Anum Anwar, ${ }^{1}$ Hina Nabi Ahmed, ${ }^{2}$ Lala Rukh Bangash, ${ }^{3}$ Farah Arshad, ${ }^{4}$ Sahira Nawaz, ${ }^{5}$ Farida Sohail ${ }^{6}$}

\begin{abstract}
Objective: To compare mean pain score of bilateral TAP block versus infiltration of local anaesthetic into surgical wound on for emergency laparotomies.

Method: Randomized control trial. Emergency Operation Theatre in Department of Anaesthesiology at Mayo Hospital, Lahore from 1st April 2016 to 31 st October 2016. Laparotomy 150 patients were arbitrarily allocated two groups Group T (receiving bilateral TAP block) and Group L (local infiltration) by random number table method after informed consent. TAP block was administered bilaterally by using $20 \mathrm{ml}$ of $0.25 \%$ bupivacaine in group $\mathrm{T}$ using a 21 -gauge needle with the help of "Double Pop Technique" at midaxillary point at height of umbilicus. While in group L surgical incision site was injected with $20 \mathrm{ml}$ of $0.25 \%$ bupivacaine immediately after closure of skin. Pain was evaluated by Visual analogue scale (VAS) at 6-hour interval postoperatively. Results were statistically analysed using SPSS version 20.0 and t-test was applied to compare mean pain score of two groups.

Results: Mean pain score in patients receiving bilateral TAP block $3.000 \pm 0.717$ were significantly reduced ( $p$ value 0.003 ) versus mean scores in patients receiving infiltration of local anaesthetic into surgical wound in emergency laparotomies $6.08 \pm 1.171$.

Conclusion: Bilateral TAP block reduced post-operative pain significantly in patients undergone emergency laparotomy.

Key Words: Emergency laparotomy, TAP block, local anaesthetic, bupivacaine, post-operative pain, analgesia.

How to Cite: Anwar A, Nabi H.A, Bangash L.R, Arshad F, Nawaz S. Comparison of analgesic efficacy of transversus abdominis plane block versus infiltration of local anaesthetic into surgical wound in emergency laparotomies: a randomized control trial. Esculapio.2020;16(04):8-13.

DOI: https://doi.org/10.51273/esc20.251642
\end{abstract}

\section{Introduction}

$\mathrm{E}$ ffective pain relief is required in patients who have undergone exploratory laparotomy since pain has many adverse effects on various systems of the body. ${ }^{1}$ Respiratory complications are much more common due to inadequate post-operative analgesia after major abdominal surgery leading to inadequate respiratory effort that results in basal atelectasis and

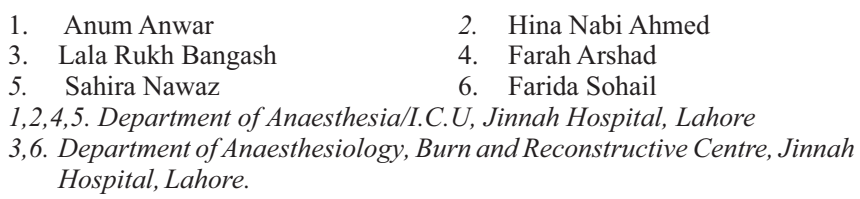

Correspondence:

Dr. Anum Anwar, Department of Anaesthesia/I.C.U, Jinnah Hospital, Lahore,E-mail: anumanwar25@gmail.com retention of secretions. ${ }^{11,12}$ Post-operative pain if not addressed properly it may lead to delayed ambulation resulting in increased chances of deep venous thromboembolism. ${ }^{2}$ Anxiety and distress related to pain affect quality of life of patient. Prolong hospital stays due to inadequate post-operative analgesia leads to financial burdens. ${ }^{13}$ Thus, adequate post-operative analgesia leads to prompt ambulation, increases comfort level of the patient and helps him to return to regular routine of life earlier. ${ }^{14}$

Multimodal analgesia is considered as an efficient way to cater post-surgical pain and in reducing adverse effects associated with heavy doses of opioids and NSAID's. ${ }^{3}$ With recent advancement in technology regional nerve blocks are gaining popularity. As component of multimodal analgesia, they reduce dosage requirements of analgesic drugs and many untoward side effects associated with them. ${ }^{15}$ Along 
with peripheral nerve blocks administration of local anaesthetic into surgical wound is an ancient technique that is still in use by numerous surgeons and anaesthetists. There are various peripheral nerve blocks that have been used to reduce pain post operatively but TAP block has been found very effective in mitigating pain after major abdominal surgeries. ${ }^{10,17}$ The anterior abdominal wall is supplied by dermatomes from T6 to L1 and TAP block numbs those nerves. This block was originally elaborated in the "lumbar triangle of Petit" in 2001 by Rafi by using conventional landmarks. Local anaesthetic is inoculated in the fascial plane that exists between two muscles transverse abdominis muscle and internal oblique muscle through which these sensory nerves pass. TAP block is an efficient analgesic method with additional benefit of intact motor and autonomic control in all abdominal procedures and permits early mobilization. ${ }^{4,16,18}$ Parasa et al piloted an analysis in which efficiency of TAP block was evaluated post operatively after open abdominal surgeries. They found pain was less intense in patients receiving TAP block $(48.07 \pm 6.76)$ in comparison to those patients who don't get block $(62.63 \pm 6.66)$. They also concluded that this results in $36 \%$ reduction in entire tramadol intake in TAP block group. Thus, TAP block being a component of multi-modal analgesia has effective part in reducing post-surgical pain and doses of other analgesic drugs. ${ }^{5,19}$ Though there were multiple studies available that prove pain-relieving efficacy ${ }^{9}$ of TAP block but there were no studies available for comparison of mean pain score of transversus abdominis plane block versus infiltration of local anaesthetic into surgical wound in emergency laparotomies. So in this trial we compared effectiveness of bilateral TAP block with infiltration of local anaesthetic into surgical wound in emergency laparotomies. This study would provide baseline data for local population and would help to mitigate postoperative pain in patients undergoing emergency laparotomies.

\section{Methods}

After endorsement from Ethical committee, randomized control trial was performed at Emergency department of Mayo Hospital, Lahore. Patients were included with non-probability consecutive sampling. Patients of either gender between 20 to 80 years, ASA I,II,III scheduled for emergency laparotomy for vis- cous perforation were included. Sample size of 150 cases (75 in each group) was calculated with $95 \%$ confidence interval, $80 \%$ power of test and taking expected mean \pm standard deviation of mean pain scores in both groups at 6 hour post-operatively with $3.33 \pm 1.51$ in TAP block group versus $5.67 \pm 1.31$ in control group in patients undergoing emergency laparotomies. ${ }^{5}$ Patients with established allergy to the study drug, with past account of opioid use or intolerance, obese (BMI $>40)$, known bleeding disorder or renal or hepatic disease, known psychiatric disorder, infection at site of injection were excluded from the study. Informed consent was obtained. Brief history was taken and related physical examination was performed. Investigations (complete blood count, platelet count, coagulation screening, and renal and liver function tests) were analysed. Patients were allocated randomly to Group $\mathrm{T}$ (receiving bilateral TAP block group) or Group L (local infiltration group) by using random number table in accordance with division ratio of $1: 1$. After shifting the patient to operating table, monitoring devices that includes pulse oximetry, electro-cardiographic monitoring and non-invasive blood pressure (NIBP) were attached and base line readings were obtained. Two large intravenous line were secured. Anaesthesia was induced with rapid sequence induction technique using injection propofol $(2-3 \mathrm{mg} / \mathrm{kg}$ ) and injection succinylcholine $(1.5 \mathrm{mg} / \mathrm{kg})$. For analgesia injection nalbuphine $(0.1 \mathrm{mg} / \mathrm{kg})$ was given just before incision. Anaesthesia was continued with isoflurane and $50 \%$ nitrous oxide in concentration of $50 \%$ in oxygen according to need of the patient.

After completion of surgery for patients with viscous perforation, just before extubation, TAP block is performed with blunted 21-gauge needle in group $\mathrm{T}$ using "double pop technique" in supine position of patient by single administrator experienced in blind technique of TAP block. In above mentioned technique the skin and subcutaneous tissue was penetrated in midaxillary plane at the height of umbilicus at right angle to the skin till resistance is felt. The needle was forced slightly through this resistance till it penetrated the external oblique aponeurosis. With slight further penetration second loss of resistance could be appreciated as needle passed through aponeurosis of internal oblique. To check the desired plane $1 \mathrm{ml}$ of drug was injected after careful aspiration. The feeling of considerable resistance on injecting drug indicated 
misplaced needle tip obviating the need to reposition it. After readjustment $20 \mathrm{ml}$ of $0.25 \%$ bupivacaine was infiltrated. The same method was used to administer block on other side. While in group L surgical incision site was injected with $20 \mathrm{ml}$ of $0.5 \%$ bupivacaine before skin closure by surgeon. In both groups dose did not exceed more than $1 \mathrm{mg} / \mathrm{kg} /$ side of $0.5 \%$ bupivacaine.

All patients were extubated and transferred to the postoperative ward. Patients were shown and instructed about VAS in the preoperative period. One end of which was marked as ' 0 ' representing no pain and the other one as ' 10 ' signifying severe most pain. Patients were requested to highlight the value that best contemplated their pain. Postoperative pain was measured by VAS at 6-hour interim post-operatively and pain scores were collected. Inj Tramadol $50 \mathrm{mg}$ was administered as rescue analgesia if VAS was more

Table 1: Frequency Distribution of Asa Grading

\begin{tabular}{lcc}
\hline \multicolumn{1}{c}{ ASA } & Frequency & Percent \\
\hline ASA II & 10 & $6.7 \%$ \\
ASA III & 134 & $89.3 \%$ \\
ASA IV & 6 & $4.0 \%$ \\
Total & 150 & $100.0 \%$ \\
\hline
\end{tabular}

Table 2: Frequency Distribution of Gender Between Both Groups

\begin{tabular}{ccccc}
\hline \multirow{2}{*}{ Gender } & $\begin{array}{c}\text { Count/ } \\
\text { percentage }\end{array}$ & \multicolumn{2}{c}{ Study groups } & \\
\cline { 3 - 4 } Male & Local & TAP & Total \\
& Count & 56 & 54 & 110 \\
& $\%$ & $51 \%$ & $49 \%$ & \\
Female & Count & 19 & 21 & 40 \\
& $\%$ & $47.5 \%$ & $52.5 \%$ & \\
\hline
\end{tabular}

Table 3:Mean Age of The Patients with Standard Deviation

\begin{tabular}{lccc|}
\multicolumn{1}{c}{ Study groups } & N & Mean & Std. Deviation \\
\hline LOCAL INFILTRATION & 75 & 57.71 & 10.686 \\
TAP BLOCK & 75 & 56.85 & 8.725 \\
\hline
\end{tabular}

Table 4: Comparison of Mean Pain Scores of The Patient

\begin{tabular}{lcccc|}
\hline \multicolumn{1}{c}{ Study groups } & N & Mean & $\begin{array}{c}\text { Std. } \\
\text { Deviation }\end{array}$ & $\begin{array}{c}\text { P - } \\
\text { value }\end{array}$ \\
\hline LOCAL INFILTRATION & 75 & 6.08 & 1.171 & 0.003 \\
TAP BLOCK & 75 & 3.00 & 0.717 & \\
\hline
\end{tabular}

There was statistically significant difference between MEAN PAIN SCORES of two study groups

than 5 at 6 hour interval postoperatively. Designated proforma was used to collect all data.

All statistical data was recorded and analysed in SPSS version 20.0. Data was stratified for age, gender, ASA grading and mean pain score 6 hour post-operatively to deal with effect modifiers. Post-stratification t-test was applied.

\section{Results}

In this study 150 patients that underwent emergency laparotomies were studies. There were no patients that were excluded from study. There were 10 ASA II patients, 134 ASA III and 6 ASA IV patients. (Table 2) Out of these 110 were males and 40 were females. (Table 3)

Mean age in TAP block group patients was $56.85 \pm$ 8.725 and mean age in patients receiving local anaesthetic infiltration into surgical wound was $57.71 \pm$ 10.686. Mean pain score in TAP block group was $3.00 \pm 0.717$ and mean pain score in patients getting local anaes-thetic infiltration into surgical wound was $6.08 \pm 1.171$. This difference in mean pain score was significant as p-value is 0.003 ( $\mathrm{p}$-value $<0.005)$.

\section{Discussion}

The result of our study shows there is considerable reduction in mean pain scores in TAP block group in comparison to patients receiving infiltration of local anaesthetics. Mean pain scores in patients with TAP block were $3.000 \pm 0.717$ as compared to group of patients receiving local anaesthetic infiltration $6.08 \pm$ 1.171 with $\mathrm{p}$ value 0.003 . Thus, TAP block causes more reduction in pain scores on VAS scale when compared to infiltration of local anaesthetic into surgical wound in emergency laparotomies. Paras et al. conducted a randomized control trial they include 60 patients from ASA state I to III that under-went emergency laparotomy. They administer TAP block before surgery in lumbar triangle of "Petit". They divided patients into two groups; one received $25 \mathrm{ml}$ of $0.25 \%$ bupivacaine and other got normal saline administered bilaterally. For post-operative pain tramadol was used along with intramuscular diclofenac sodium. Tramadol was administered with PCA (patient controlled analgesic) pump while dose of diclofenac sodium adjusted 12-hourly. The tramadol requirement at 2 hour and total given in 24 hours was measured along with assessment of pain and side effects of opioids. They found that TAP block group had mean pain scores $48.07 \pm 6.76$ with total tramadol intake in 24 hours $281.33 \pm 69.66 \mathrm{mg}$ when compared to the control group having mean scores for pain 
$62.63 \pm 6.66$ and tramadol intake $439 \pm 68.59 \mathrm{mg}$. Thus, our study is in consistency with the fact that TAP block significantly reduces mean pain scores but, in our study, comparison is made between TAP block and local anaesthetic infiltration. Similarly Vijayalakshmi et al. conducted study in patients undergoing gynaecological procedures in which open abdominal surgical approach used. ${ }^{6} \mathrm{He}$ randomly allocated patients into group receiving TAP block and other group receiving local anaesthesia into surgical site. He administered $0.25 \%$ bupivacaine at dose of $0.6 \mathrm{ml} / \mathrm{kg}$ bilaterally at height of umbilicus in triangle of "petit" after completion of surgery while other group got infiltration of local anaesthetic into surgical lesion just before extubation after placement of skin sutures. They recorded time at which patient demanded rescue analgesia and monitored intensity of pain at that moment by using VAS scale. They used morphine as rescue analgesia at dose of $0.1 \mathrm{mg} / \mathrm{kg}$ and it was continuously administered thereafter with PCA pump for 24 hours. Total amount of morphine consumed in period of 24 hours post operatively calculated. They concluded that In Group T patients demanded foremost dosage of morphine considerably later and at that moment pain was not much intense as compared to other group $(\mathrm{P}=0.001$ and 0.003 respectively). Total intake of morphine in group receiving local anaesthetic was more as compared to TAP block group so the side effects of morphine also predominated in this group. Thus conclusion of their study was that transversus abdominis plane block is an effective modality to reduce pain with few adverse effects and their results are in line with our conclusion but we have done comparative study in patients undergoing exploratory laparotomy. Likewise, Priya et al conducted study in 60 patients who underwent major abdominal operations. They calculated doses of tramadol required post opera-tively at 24 hour time interval and at 48 hour time interval. According to their study, total amount of tramadol consumed in patients in TAP block in 24 hour time duration $(210.05 \pm 20.5$ vs. $320.05 \pm 10.6 ; \mathrm{P}<0.01)$ and at 48 hour interval ( $508.25 \pm 20.6$ vs. $550.25 \pm 20.6$; $\mathrm{P}<$ $0.01)$. They also concluded that time at which patient demanded first dose of analgesic drug was more as compared to other group (178.5 \pm 45.6 vs. $23.5 \pm 3.8$; $\mathrm{P}<0.001)$. Pain intensity measured at different intervals in 24 hours period post operatively. Pain scores were significantly less in TAP block group contrary to other group. ${ }^{7}$ Thus, TAP block is very effective modality with crucial role in mitigation of pain post operatively. The results of our study add significance to above mentioned study and prove it to be more effective as compared to ancient technique of wound infiltration with local anaesthetic. Ejas et al. performed randomized control trial in patients who underwent single incision laparoscopic cholecystectomy. ${ }^{8}$ They performed TAP block under USG guidance with ropivacaine in concenteration of $0.375 \%$ bilaterally in mid axillary plane in one group while other group received local anaesthetic infiltration at site of insertion of ports. They calculated amount of morphine required post operatively in 24 hours. Morphine requirement in group receiving TAP block was $34.57+14.67 \mathrm{mg}$ as compared to other group in which values were $32.76+14.34 \mathrm{mg}$. They found TAP block did not cause significant reduction in post-operative analgesic requirement as compared to conventional methods. This is contrary to our result in which we found TAP block more effective. There are certain limitations of our study that it does not measure time of administration of first dose of analgesia, total dosage requirements of analgesia 24 hour post-operatively and incidence of nausea and vomiting.

\section{Conclusion}

Outcome of our study shows substantial reduction in mean pain scores after bilateral TAP block infiltration in patients with emergency laparotomy. Mean pain scores remain less than 4 on VAS pain scale in patients with TAP block. Hence it is concluded that TAP block significantly reduces pains score on VAS scale as compared to surgical wound infiltration with local anaesthetic in patients with emergency laparotomies. TAP block is better modality as part of multimodal analgesia to reduce post-operative pain as compared to surgical wound infiltration with local anaesthetic.

\section{Conflict of Interest: $\quad$ None}

\section{References}

1. Dal Moro F, Pavarin P, Mangano A, Aiello L, Valotto C, Zattoni F. Mp47-01 Ultrasound-Guided Trans- 
versus Abdominis Plane (Tap) Block For RobotAssisted Radical Prostatectomy. The Journal of Urology. 2017 Apr 1; 197(4):e628 doi: 10.1007/s11701018-0858-6.

2. Gao T, Zhang JJ, Xi FC, Shi JL, Lu Y, Tan SJ, Yu WK. Evaluation of transversus abdominis plane (TAP) block in hernia surgery: a meta-analysis. The Clinical journal of pain. 2017 Apr 1;33(4):369-75. doi: 10. 1097/AJP.0000000000000412.

3. Komatsu S, Fujiwara Y. Transversus abdominis plane block in laparoscopic colorectal surgery. Annals of Laparoscopic and Endoscopic Surgery. 2017 Mar 17;2(1) doi: 10.21037/ales.2017.03.08

4. Walter CJ, Maxwell-Armstrong C, Pinkney TD, Conaghan PJ, Bedforth N, Gornall CB, Acheson AG . A randomised controlled trial of the efficacy of ultrasound-guided transversus abdominis plane (TAP) block in laparoscopic colorectal surgery. Surg Endosc 2013;27:2366-72 doi: 10.1007/s00464-013-2791-0.

5. Mrunalini P, Rama NVR, Nath VN, Saheb SM. Efficacy of transversus abdominis plane block in patients undergoing emergency laparotomies. Anesth Essays Res 2014;8:377-82. DOI: 10.4103/02591162. 143153

6. Sivapurapu V, Vasudevan A, Gupta S, Badhe AS. Comparison of analgesic efficacy of transversus abdominis plane block with direct infiltration of local anesthetic into surgical incision in lower abdominal gynecological surgeries. J Anaesthesiol Clin Pharmacol 2013;29:71-5 DOI: 10.4103/0970-9185.105807.

7. Saxena A, Bansal R, Mittal A, Shrivastava U, Sharma P, Chand T. Evaluation Of Postoperative Analgesic Efficacy Of Transversus Abdominis Plane Block After Abdominal Surgery: A Comparative Study. Journal of Natural Science, Biology and Medicine 2013; 4(1):177. DOI: 10.4103/0976-9668.107286

8. Bava EP, Ramachandran R, Rewari V. Analgesic efficacy of ultrasound guided transversus abdominis plane block versus local anesthetic infiltration in adult patients undergoing single incision laparoscopic cholecystectomy: A randomized controlled trial. Anesthesia, essays and researches. 2016 Sep; 10(3): 561. doi: 10.4103/0259-1162.186620

9. Dai C, Zhang K, Huang J. The Efficacy of Transversus Abdominis Plane Block for Abdominal Hysterectomy Post-operative Analgesia. Cureus. 2018; 10(8): e3131 http://doi:10.7759/cureus.3131

10. Peltrini, R., Cantoni, V., Green, R. et al. Efficacy of transversus abdominis plane (TAP) block in colorectal surgery: a systematic review and meta-analysis. Tech Coloproctol 24, 787-802 (2020). https:// doi. org/ 10.1007/s10151-020-02206-

11. Patch III RK, Eldrige JS, Moeschler SM, Pingree MJ. Dexmedetomidine as part of a multimodal analgesic treatment regimen for opioid induced hyperalgesia in a patient with significant opioid tolerance. Case Reports in Anesthesiology. 2017 Jan 1;2017. https:// doi.org/10.1155/2017/9876306

12. Babu S, Gupta BK, Gautam GK. A comparative study for post operative analgesia in the emergency laparotomies: Thoracic epidural ropivacaine with nalbuphine and ropivacaine with butorphanol. Anesthesia, essays and researches. 2017 Jan;11(1):155. https:// dx. doi.org/10.4103\%2F0259-1162.186593

13. Martin LD, Adams TL, Duling LC, Grigg EB, Bosenberg A, Onchiri F, Jimenez N. Comparison between epidural and opioid analgesia for infants undergoing major abdominal surgery. Pediatric Anesthesia. 2019 Aug;29(8):835-42. https:// doi. org/ 10.1111/pan.13672

14. Toro MM, John S, Faruqui AR. Pattern of use of analgesics in post-operative pain management in adults undergoing laparotomy surgery: a prospective observational study. International Surgery Journal. 2018 Jan 25;5(2):662-7. https://dx.doi.org/10.18203/ 2349-2902.isj20180372

15. Bang S, Chung J, Kwon W, Yoo S, Soh H, Lee SM. Erector spinae plane block for multimodal analgesia after wide midline laparotomy: A case report. Medicine. 2019 May;98(20). https://dx.doi.org/10. 1097\% 2FMD.0000000000015654

16. Baytar Ç, Y1lmaz C, Karasu D, Topal S. Comparison of ultrasound-guided subcostal transversus abdominis plane block and quadratus lumborum block in laparoscopic cholecystectomy: a prospective, randomized, controlled clinical study. Pain Research and Management. 2019 Feb 3;2019. https://doi.org/10. $1155 / 2019 / 2815301$

17. Yoshida T, Furutani K, Watanabe Y, Ohashi N, Baba $\mathrm{H}$. Analgesic efficacy of bilateral continuous transversus abdominis plane blocks using an oblique subcostal approach in patients undergoing laparotomy for gynaecological cancer: a prospective, randomized, triple-blind, placebo-controlled study. BJA: British Journal of Anaesthesia. 2016 Dec 12;117(6):81220. https://doi.org/10.1093/bja/aew339

18. Chang H, Rimel BJ, Li AJ, Cass I, Karlan BY, Walsh C. Ultrasound guided transversus abdominis plane (TAP) block utilization in multimodal pain management after open gynecologic surgery. Gynecologic oncology reports. 2018 Nov 1;26:75-7. https:// doi. org/10.1016/j.gore.2018.10.007 
19. Arafa As, Alshal Sm, Mohamed AA, Mansour MA, Turki DA. Ultrasound Guided Transversus Abdominis Plane Block Combined with Transversalis Fascia Plane Block Versus Ultrasound Guided Transversus Abdominis Plane Block Alone for Pain Relief in Patients Undergoing Laparotomy.

\section{Author's Contribution}

AA: Concept, Conduction of study, manuscript preparation and editing, literature review

HNA: Manuscript preparation, proof reading and critically reviewed the article

LRB: Statistical analysis of the data

SNK: Data collection

FA: Data collection 